Geometry 85 Topology

Volume 6 (2002) 905-916

Published: 31 December 2002

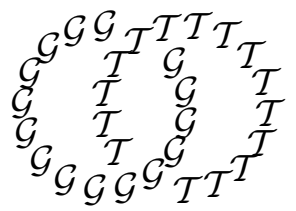

\title{
Volume change under drilling
}

\author{
IAN AGOL \\ MSCS, SEO 322, m/c 249, University of Illinois at Chicago \\ 851 S Morgan St, Chicago, IL 60607-7045, USA \\ Email: agol@math.uic.edu \\ URL: http://www.math.uic.edu/ ${ }^{\sim}$ agol
}

\begin{abstract}
Given a hyperbolic 3-manifold $M$ containing an embedded closed geodesic, we estimate the volume of a complete hyperbolic metric on the complement of the geodesic in terms of the geometry of $M$. As a corollary, we show that the smallest volume orientable hyperbolic 3 -manifold has volume $>.32$.
\end{abstract}

AMS Classification numbers Primary: 57M50

Secondary: $53 \mathrm{C} 15,53 \mathrm{C} 22$

Keywords: Hyperbolic structure, 3-manifold, volume, geodesic

Proposed: David Gabai

Seconded: Walter Neumann, John Morgan
Received: 17 January 2001

Revised: 7 November 2002

(c) Geometry $8 \mathcal{G}$ Topology $\mathcal{P}$ ublications 


\section{Introduction}

Kerckhoff showed that given a hyperbolic 3-manifold $M$ and an embedded geodesic $\gamma \subset M$, the manifold $M-\gamma \equiv M_{\gamma}$ has a metric of negative sectional curvature [11], using a method of Gromov and Thurston [2]. Therefore by Thurston, $M_{\gamma}$ admits a unique complete hyperbolic metric, ie, a metric of constant sectional curvature -1 [13]. In theorem 2.1 of this paper, we show that if $\gamma$ has an embedded tubular neighborhood of radius $R$, then $\operatorname{Vol}\left(M_{\gamma}\right) \leq(\operatorname{coth} R)^{\frac{5}{2}}(\operatorname{coth} 2 R)^{\frac{1}{2}} \operatorname{Vol}(M)$, where Vol denotes the volume of the unique complete hyperbolic structure. Specifically, we use Kerckhoff's method to fill in a metric on $M_{\gamma}$ in order to apply a theorem of Boland, Connell, and Souto [3], which is based on an important technique of Besson, Courtois, and Gallot [1], to get an upper bound on the hyperbolic volume of $M_{\gamma}$ in terms of the geometry of $M$. As a corollary, we show that the smallest volume orientable hyperbolic $3-$ manifold has volume $>.32$. It is conjectured that the Weeks manifold, which has volume $=.9427 \ldots$, is the smallest volume hyperbolic 3-manifold. This is known to be the smallest volume arithmetic hyperbolic 3-manifold, by a result of Chinburg, Friedmann, Jones, and Reid [6]. The smallest volume orientable cusped manifolds are known to be the figure eight knot complement and its sibling, which have volume $=2.0298 \ldots$, by a result of Cao and Meyerhoff [5], which we use combined with the main theorem to obtain our lower bound.

Acknowledgements We thank Bill Thurston for helpful conversations, in particular for explaining lemma 2.3. This research was mostly carried out at the University of Melbourne, and we thank Iain Aitchison, Hyam Rubinstein and the maths department for their hospitality. We thank Oliver Goodman for help using his program tube [10]. We thank the referees for many useful comments, and for finding a mistake in an earlier version. The author was partially supported by ARC grant 420998 .

\section{Estimates}

Theorem 2.1 Let $M$ be an orientable hyperbolic 3-manifold with metric $\nu$. Let $\gamma \subset M$ be a geodesic in $M$ of length $l$ with an embedded open tubular neighborhood $C$ of radius $R$, and with complete hyperbolic metric $\tau$ on $M_{\gamma}$.

Then $\operatorname{Vol}\left(M_{\gamma}, \tau\right) \leq(\operatorname{coth} R \operatorname{coth} 2 R)^{\frac{3}{2}}\left(\operatorname{Vol}(M, \nu)+\pi l \sinh ^{2} R\left(\frac{\operatorname{coth} R}{\operatorname{coth} 2 R}-1\right)\right)$

$$
\leq(\operatorname{coth} R)^{\frac{5}{2}}(\operatorname{coth} 2 R)^{\frac{1}{2}} \operatorname{Vol}(M, \nu) .
$$


Remark This theorem can be easily generalized to a geodesic link in $M$ with an embedded tubular neighborhood, but we restrict to the case of one component to simplify the argument, since this is the context of our main application. Also, if $M$ is non-orientable, then we may apply this theorem to the 2 -fold orientable covering to recover the same estimate.

The intuition behind the estimate is that we construct a Riemannian metric $\rho$ on $M_{\gamma}$ which coincides with the metric $\nu$ on $M \backslash C$, such that $\operatorname{Ric}_{\rho} \geq-2 k \rho$, where $k=\operatorname{coth} R \operatorname{coth} 2 R$. Then we would like to apply the following Volume Theorem of Boland, Connell and Souto to compare the two metrics on $M_{\gamma}$ :

Theorem 2.2 [3] Let $(M, g)$ and $\left(M_{0}, g_{0}\right)$ be two oriented complete finite volume Riemannian manifolds of the same dimension $\geq 3$, and suppose that Ric $_{g} \geq-(n-1) g$, and $-a \leq K_{g_{0}} \leq-1$. Then for all proper continuous maps $f: M \rightarrow M_{0}$,

$$
\operatorname{Vol}(M, g) \geq|\operatorname{deg}(f)| \operatorname{Vol}\left(M_{0}, g_{0}\right)
$$

and equality holds if and only if $f$ is proper homotopic to a Riemannian covering.

In our situation, we want to choose $\left(M_{0}, g_{0}\right)=\left(M_{\gamma}, \tau\right)$, where $K_{\tau}=-1$, since the metric is hyperbolic, $(M, g)=\left(M_{\gamma}, k \rho\right)$, where we scale $\rho$ by $k$ so that it satisfies $R i c_{k \rho} \geq-2 k \rho$ almost everywhere, and $f=I d_{M_{\gamma}}$ is degree one and proper. Then we would like to apply theorem 2.2 to conclude that $\operatorname{Vol}\left(M_{\gamma}, k \rho\right) \geq \operatorname{Vol}\left(M_{\gamma}, \tau\right)$, which may be computed to obtain the estimate of theorem 2.1. Some technicalities are involved in that the metric $\rho$ we construct is only $C^{1}$, so that we must approximate $\rho$ by smooth metrics in order to apply theorem 2.2 .

Proof of theorem 2.1 The tubular neighborhood $C$ of radius $R$ of $\gamma$ of length $l$ has a metric in cylindrical coordinates $(r, \theta, \lambda)$ given by $d s^{2}=d r^{2}+$ $\sinh ^{2} r d \theta^{2}+\cosh ^{2} r d \lambda^{2}$, on the cylinder $C=[0, R] \times[0,2 \pi] \times[0, l]$ with $(r, 0, \lambda) \sim$ $(r, 2 \pi, \lambda),(r, \theta, 0) \sim(r, \theta+\phi, l)$ and $(0, \theta, \lambda) \sim(0,0, \lambda)$, for some constant $\phi$ giving the rotational part of the holonomy of $\gamma . M_{\gamma}$ may be obtained from $M$ by removing $C$ from $M$ and replacing it with the region $C^{\prime}=$ $(-\infty, R] \times[0,2 \pi] \times[0, l]$, with the same first two identifications as above. We will give $C^{\prime}$ a metric of the form $d s^{2}=d r^{2}+f(r)^{2} d \theta^{2}+g(r)^{2} d \lambda^{2}$. These types of metrics were considered by Gromov and Thurston in proving their $2 \pi$-theorem for obtaining negatively curved metrics on Dehn filled 3-manifolds [2]. We need to compute sectional curvatures for this metric. This has been done by Bleiler and Hodgson [2], but we will give a sketch of a derivation as explained to us by Thurston. 
Lemma 2.3 The metric $d r^{2}+f(r)^{2} d \theta^{2}+g(r)^{2} d \lambda^{2}$ on $\mathbb{R}^{3}$ has sectional curvatures

$$
K_{\theta \lambda}=-\frac{f^{\prime} g^{\prime}}{f g}, K_{r \theta}=-\frac{f^{\prime \prime}}{f}, K_{r \lambda}=-\frac{g^{\prime \prime}}{g}
$$

Proof First we will compute $K_{\theta \lambda}$. Fix a reference point $\left(r_{0}, \theta_{0}, \lambda_{0}\right)$ at which to compute the curvature. Consider the plane $H=\left(r_{0}, \theta, \lambda\right)$. $H$ is isometric to the euclidean plane, so the Gauss curvature of $H$ is 0 . The reflection through the plane $\theta=\theta_{0}$ preserves $H$ and $\left(r_{0}, \theta_{0}, \lambda_{0}\right)$, so the lines of principal curvature of $H$ at $\left(r_{0}, \theta_{0}, \lambda_{0}\right)$ are invariant under this reflection. Thus, they must be parallel to the vectors $\frac{\partial}{\partial \theta}$ and $\frac{\partial}{\partial \lambda}$. To compute the principal curvatures, we can compute the geodesic curvatures of $H$-geodesics in the $\theta$ and $\lambda$ directions. Consider the curve $c=\left(r_{0}, \theta, \lambda_{0}\right)$. The length of $c$ on the interval $\left[\theta_{0}-\epsilon, \theta_{0}+\epsilon\right]$ is $2 \epsilon f\left(r_{0}\right)$. If we do a variation of this curve normal to $H$, the length is $2 \epsilon f(r)$. Since the reflection in the plane $\lambda=\lambda_{0}$ preserves $c$, the osculating plane of $c$ must be the plane $\lambda=\lambda_{0}$, so that the normal variation lies in this osculating plane. The geodesic curvature is then the logarithmic derivative of the length of the variation (compare to the euclidean case), $k_{g}=\frac{\partial}{\partial r} \log (2 \epsilon f(r))=\frac{f^{\prime}}{f}$, which gives the principal curvature of $H$ in the direction of $\theta$. The other principal curvature is $\frac{g^{\prime}}{g}$. Thus, the Gauss curvature of $H$ is $0=K_{\theta \lambda}+\frac{f^{\prime} g^{\prime}}{f g}$, so $K_{\theta \lambda}=-\frac{f^{\prime} g^{\prime}}{f g}$.

To compute $K_{r \theta}$, notice that the plane $\lambda=\lambda_{0}$ is geodesic. So the sectional curvature is the Gauss curvature of the plane $\lambda=\lambda_{0}$. Consider the annulus $S_{r}=\left[r_{0}, r\right] \times[0,2 \pi] \times\left\{\lambda_{0}\right\}$. By the Gauss-Bonnet theorem,

$$
\int_{S_{r}} K_{r \theta} d A+\int_{\partial S_{r}} k_{g} d s=2 \pi \chi\left(S_{r}\right)=0
$$

We have

$$
\begin{aligned}
& \frac{\partial}{\partial r} \int_{S_{r}} K_{r \theta} d A=\frac{\partial}{\partial r} \int_{r_{0}}^{r} \int_{0}^{2 \pi} K_{r \theta} f(r) d \theta d r=2 \pi K_{r \theta} f(r) \\
& =-\frac{\partial}{\partial r} \int_{\partial S_{r}} k_{g} d s=-\frac{\partial}{\partial r} \int_{0}^{2 \pi} \frac{f^{\prime}(r)}{f(r)} f(r) d \theta=-2 \pi f^{\prime \prime}(r) .
\end{aligned}
$$

Thus $K_{r \theta}=-\frac{f^{\prime \prime}}{f}$. Similarly, $K_{r \lambda}=-\frac{g^{\prime \prime}}{g}$.

Kerckhoff's proof that $M_{\gamma}$ has a metric of negative sectional curvature follows from lemma 2.3 by noting that one may choose $C^{\infty}$ functions $f(r)$ and $g(r)$ on $(-\infty, R]$ such that for $r$ near $R, f(r)=\sinh r$ and $g(r)=\cosh r$, and such 
that all of $f(r), f^{\prime}(r), f^{\prime \prime}(r), g(r), g^{\prime}(r), g^{\prime \prime}(r)$ are $>0$ (see figure 1). Then it follows that $M_{\gamma}$ is atoroidal, anannular, and irreducible, so $M_{\gamma}$ has a complete hyperbolic metric $\tau$ of finite volume by Thurston's hyperbolization theorem of Haken manifolds [13].

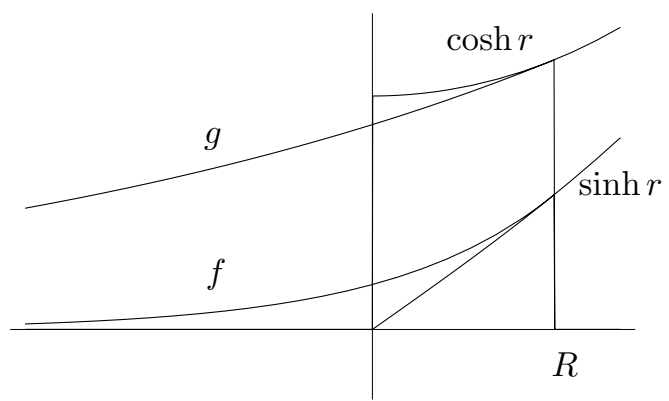

Figure 1: Constructing a negatively curved metric

We want to construct metrics on $M_{\gamma}$ for which we may apply theorem 2.2. Let $f(r)=(\sinh R) e^{(\operatorname{coth} R)(r-R)}$, and $g(r)=(\cosh R) e^{(\tanh R)(r-R)}$. This gives a $C^{\infty}$ metric on $C^{\prime}$, which induces a $C^{1}$ metric $\rho$ on $M_{\gamma}$, where $\rho$ agrees with $\nu$ on $M_{\gamma} \backslash C^{\prime}=M \backslash C$.

On $C^{\prime}$, choose the orthonormal basis for the tangent space at a fixed point $\left(e_{1}, e_{2}, e_{3}\right)=\left(\frac{\partial}{\partial r}, \frac{1}{f} \frac{\partial}{\partial \theta}, \frac{1}{g} \frac{\partial}{\partial \lambda}\right)$. The reflections $\theta \rightarrow-\theta$ and $\lambda \rightarrow-\lambda$ preserve the metric locally, so must preserve the maximal and minimal sectional curvatures. The only tangent planes preserved by both these reflections are spanned by $\left\{e_{i}, e_{j}\right\}, i \neq j$. Thus, we conclude that the sectional curvatures $K_{r \theta}=K_{e_{1} e_{2}}$, $K_{\theta \lambda}=K_{e_{2} e_{3}}$, and $K_{r \lambda}=K_{e_{1} e_{3}}$ realize the minimum and maximum sectional curvatures. It is well known that in such coordinates, the Ricci curvature is diagonalized. To see this, one computes the curvature tensor $R\left(e_{i}, e_{j}, e_{k}, e_{l}\right)$ (see [9], ch. III A, for notational conventions). The sectional curvature of the plane spanned by $\left\{e_{i}, e_{j}\right\}$ is $R\left(e_{i}, e_{j}, e_{i}, e_{j}\right)$. If this sectional curvature is maximal, then we see that

$\left.\frac{d}{d \varphi} R\left(e_{i},\left(e_{j} \cos \varphi+e_{k} \sin \varphi\right), e_{i},\left(e_{j} \cos \varphi+e_{k} \sin \varphi\right)\right)\right|_{\varphi=0}=2 R\left(e_{i}, e_{k}, e_{i}, e_{j}\right)=0$.

Similarly, one may see that $R\left(e_{j}, e_{k}, e_{j}, e_{i}\right)=R\left(e_{k}, e_{i}, e_{k}, e_{j}\right)=0$, by exchanging the roles of $i$ and $j$, and using the same observation for the minimal sectional curvature. So all the off-diagonal terms of $R$ vanish. Since $\operatorname{Ric}_{\rho}\left(e_{i}, e_{j}\right)=$ $\sum_{k=1}^{3} R\left(e_{k}, e_{i}, e_{k}, e_{j}\right)=0$, if $i \neq j$ we see that the off-diagonal terms of the Ricci tensor vanish as well. We compute $\operatorname{Ric}_{\rho}$ in the basis $\left(e_{1}, e_{2}, e_{3}\right)$ : 


$$
\begin{gathered}
R i c_{\rho}=\left[\begin{array}{ccc}
K_{r \theta}+K_{r \lambda} & 0 & 0 \\
0 & K_{r \theta}+K_{\theta \lambda} & 0 \\
0 & 0 & K_{r \lambda}+K_{\theta \lambda}
\end{array}\right] \\
=\left[\begin{array}{ccc}
-\operatorname{coth}^{2} R-\tanh ^{2} R & 0 & 0 \\
0 & -\operatorname{coth}^{2} R-1 & 0 \\
0 & 0 & -\tanh ^{2} R-1
\end{array}\right] \\
\geq-2 \operatorname{coth} R \operatorname{coth} 2 R\left[\begin{array}{lll}
1 & 0 & 0 \\
0 & 1 & 0 \\
0 & 0 & 1
\end{array}\right]=(-2 \operatorname{coth} R \operatorname{coth} 2 R) \rho
\end{gathered}
$$

by lemma 2.3. We have

$$
\begin{gathered}
\operatorname{Vol}\left(M_{\gamma}, \rho\right)=\operatorname{Vol}(M, \nu)-\operatorname{Vol}(C, \nu)+\operatorname{Vol}\left(C^{\prime}, \rho\right) \\
=\operatorname{Vol}(M, \nu)-\pi l \sinh ^{2} R+2 \pi l \frac{\sinh R \cosh R}{\operatorname{coth} R+\tanh R} \\
=\operatorname{Vol}(M, \nu)+\pi l \sinh ^{2} R\left(\frac{\operatorname{coth} R}{\operatorname{coth} 2 R}-1\right) \leq \operatorname{Vol}(M, \nu) \frac{\operatorname{coth} R}{\operatorname{coth} 2 R}
\end{gathered}
$$

where the last inequality uses the fact that $\pi l \sinh ^{2} R=\operatorname{Vol}(C, \nu) \leq \operatorname{Vol}(M, \nu)$.

Lemma 2.4 There is a family of negatively curved metrics $\rho_{\epsilon}$ on $M_{\gamma}$ such that $\operatorname{Vol}\left(M_{\gamma}, \rho_{\epsilon}\right) \rightarrow \operatorname{Vol}\left(M_{\gamma}, \rho\right)$ and $\operatorname{Ric}_{\rho_{\epsilon}} \geq-2 k_{\epsilon} \rho_{\epsilon}$, where $k_{\epsilon} \rightarrow \operatorname{coth} R \operatorname{coth} 2 R$ as $\epsilon \rightarrow 0^{+}$.

Using this lemma, we can finish the proof of theorem 2.1. Consider the family of metrics $\rho_{\epsilon}$ on $M_{\gamma}$. The metrics $k_{\epsilon} \rho_{\epsilon}$ on $M_{\gamma}$ satisfy $R i c_{k_{\epsilon} \rho_{\epsilon}}=R i c_{\rho_{\epsilon}} \geq-2 k_{\epsilon} \rho_{\epsilon}$. Given this Ricci curvature control, we can apply theorem 2.2 with $(M, g)=$ $\left(M_{\gamma}, \tau\right),\left(M_{0}, g_{0}\right)=\left(M_{\gamma}, k_{e} \rho_{\epsilon}\right)$, and $f=I d_{M_{\gamma}}$ to conclude that

$$
k_{\epsilon}^{3 / 2} \operatorname{Vol}\left(M_{\gamma}, \rho_{\epsilon}\right)=\operatorname{Vol}\left(M_{\gamma}, k_{\epsilon} \rho_{\epsilon}\right) \geq \operatorname{Vol}\left(M_{\gamma}, \tau\right) .
$$

In the limit, we have

$$
\begin{gathered}
\operatorname{Vol}\left(M_{\gamma}, \tau\right) \leq(\operatorname{coth} R \operatorname{coth} 2 R)^{3 / 2} \operatorname{Vol}\left(M_{\gamma}, \rho\right) \\
=(\operatorname{coth} R \operatorname{coth} 2 R)^{\frac{3}{2}}\left(\operatorname{Vol}(M, \nu)+\pi l \sinh ^{2} R\left(\frac{\operatorname{coth} R}{\operatorname{coth} 2 R}-1\right)\right) \\
\leq(\operatorname{coth} R)^{5 / 2}(\operatorname{coth} 2 R)^{1 / 2} \operatorname{Vol}(M, \nu) .
\end{gathered}
$$

To complete the proof of theorem 2.1, we need to prove lemma 2.4 . 
Proof of lemma 2.4 We will construct the metric $\rho_{\epsilon}$ by smoothing $\rho$ on the region $C^{\prime}$, by smoothing the functions $f(r)$ and $g(r)$ using the construction given in the following lemma.

\section{Lemma 2.5 Suppose}

$$
a(r)= \begin{cases}b(r) & r<R, \\ c(r) & r \geq R,\end{cases}
$$

where $b(r)$ and $c(r)$ are $C^{\infty}$ on $(-\infty, \infty)$, and $b(R)=c(R), b^{\prime}(R)=c^{\prime}(R)$. Then we may find $C^{\infty}$ functions $a_{\epsilon}$ on $(-\infty, \infty)$ for $\epsilon>0$ such that

(1) there is a $\delta(\epsilon)>0$ such that $\lim _{\epsilon \rightarrow 0^{+}} \delta(\epsilon)=0$ and $a_{\epsilon}(r)=b(r)$ for $r \leq$ $R-\delta(e), a_{\epsilon}(r)=c(r)$ for $r \geq R$, and

$$
\begin{aligned}
& \min \left\{b^{\prime \prime}(R), c^{\prime \prime}(R)\right\}=\lim _{\epsilon \rightarrow 0^{+} R-\delta(\epsilon) \leq r \leq R} \inf _{\epsilon} a_{\epsilon}^{\prime \prime}(r) \\
\leq & \lim _{\epsilon \rightarrow 0^{+}} \sup _{R-\delta(\epsilon) \leq r \leq R} a_{\epsilon}^{\prime \prime}(r)=\max \left\{b^{\prime \prime}(R), c^{\prime \prime}(R)\right\} .
\end{aligned}
$$

Proof of lemma 2.5 The idea is to smoothly interpolate between the functions $b(r)$ and $c(r)$. If we were to just use a partition of unity to interpolate between them, we would not control the first and second derivatives of the interpolation. So instead, we use a partition of unity to interpolate between $b^{\prime \prime}(r)$ and $c^{\prime \prime}(r)$ to get a function $\eta_{\epsilon}^{\prime}(r)$. Integrating twice, we must add bump functions in order to get the correct properties.

Define

$$
\alpha(r)= \begin{cases}e^{-1 / r^{2}} e^{-1 /(1-r)^{2}} & 0<r<1, \\ 0 & r \leq 0, r \geq 1 .\end{cases}
$$

Then $\alpha(r)$ is a $C^{\infty}$ bump function. Let $\beta(r)=\int_{0}^{r} \alpha(t) d t / \int_{0}^{1} \alpha(t) d t$. Then $\beta^{\prime}(r) \geq 0$, and $\beta^{\prime}$ is supported on $[0,1]$. Let $\phi_{\epsilon}(r)=\beta((r-R) / \epsilon+1)$, if $\epsilon>0$, and $\phi_{0}(r)=0$. Let $\eta_{\epsilon}^{\prime}(r)=b^{\prime \prime}(r)\left(1-\phi_{\epsilon}(r)\right)+c^{\prime \prime}(r) \phi_{\epsilon}(r)$ (figure 2(a)). Then $\eta_{\epsilon}^{\prime}(r)=b^{\prime \prime}(r)$ for $r \leq R-\epsilon$, and $\eta_{\epsilon}^{\prime}(r)=c^{\prime \prime}(r)$ for $r \geq R$, and thus $\eta_{\epsilon}^{\prime}(r)$ interpolates between $b^{\prime \prime}(r)$ and $c^{\prime \prime}(r)$. Let $\eta_{\epsilon}(r)=b^{\prime}(R-\epsilon)+\int_{R-\epsilon}^{r} \eta_{\epsilon}^{\prime}(t) d t$. $\eta_{\epsilon}(r)=b^{\prime}(r)$ for $r \leq R-\epsilon$, but it will differ from $c^{\prime}(r)$ for $r \geq R$ by a constant (figure $3(\mathrm{a})$ ), so we must adjust $\eta_{\epsilon}(r)$ to obtain a function which interpolates between $b^{\prime}$ and $c^{\prime}$. Let $\iota(\epsilon)=\left|c^{\prime}(R)-\eta_{\epsilon}(R)\right|^{1 / 2}$, then $\lim _{\epsilon \rightarrow 0^{+}} \iota(\epsilon)=0$. Let $\kappa_{\epsilon}^{\prime}(r)=\eta_{\epsilon}(r)+\left(c^{\prime}(R)-\eta_{\epsilon}(R)\right) \phi_{\iota(\epsilon)}(r)$ (figure 3(a)). Now $\kappa_{\epsilon}^{\prime}(r)=c^{\prime}(r)$ for $r \geq$ $R$, and $\kappa_{\epsilon}^{\prime}(r) \rightarrow a^{\prime}(r)$ uniformly as $\epsilon \rightarrow 0^{+}$. We have $\left|c^{\prime}(R)-\eta_{\epsilon}(R)\right| \phi_{\iota(\epsilon)}^{\prime}(r)=$ $\iota(\epsilon) \beta^{\prime}((r-R) / \iota(\epsilon)+1)$, which goes to 0 uniformly as $\epsilon \rightarrow 0^{+}$, since $\beta^{\prime}$ is 
uniformly bounded. Let $\kappa_{\epsilon}(r)=b(R-\iota(\epsilon))+\int_{R-\iota(\epsilon)}^{r} \kappa_{\epsilon}^{\prime}(t) d t$, and define $\omega(\epsilon)=$ $\left|c(R)-\kappa_{\epsilon}(R)\right|^{1 / 3}$. Let $a_{\epsilon}(r)=\kappa_{\epsilon}(r)+\left(c(R)-\kappa_{\epsilon}(R)\right) \phi_{\omega(\epsilon)}(r)$ (figure $3(\mathrm{~b})$ ). We choose $\omega(\epsilon)$ so that the bump function has small enough second derivative, that is $\left|c(R)-\kappa_{\epsilon}(R)\right| \phi_{\omega(\epsilon)}^{\prime \prime}(r)=\omega(\epsilon) \beta^{\prime \prime}((r-R) / \omega(\epsilon)+1) \rightarrow 0$ as $\epsilon \rightarrow 0^{+}$. Let $\delta(\epsilon)=\max \{\epsilon, \iota(\epsilon), \omega(\epsilon)\}$. It is clear from the construction that $a_{\epsilon}(r)=b(r)$, for $r \leq R-\delta(\epsilon)$, and $a_{\epsilon}(r)=c(r)$, for $r \geq R$. We also have $\min \left\{b^{\prime \prime}(R), c^{\prime \prime}(R)\right\}=$ $\lim _{\epsilon \rightarrow 0^{+} R-\delta(\epsilon)<r<R} \inf _{\epsilon}^{\prime}(r) \leq \lim _{\epsilon \rightarrow 0^{+}} \sup _{R-\delta(\epsilon)<r<R} \eta_{\epsilon}^{\prime}(r)=\max \left\{b^{\prime \prime}(R), c^{\prime \prime}(R)\right\}$. Now, since $\left|a_{\epsilon}^{\prime \prime}(r)-\eta_{\epsilon}^{\prime}(r)\right| \leq\left|\iota(\epsilon) \beta^{\prime}((r-R) / \iota(\epsilon)+1)\right|+\left|\omega(\epsilon) \beta^{\prime \prime}((r-R) / \omega(\epsilon)+1)\right| \rightarrow 0$ as $\epsilon \rightarrow 0^{+}$(figure $2(\mathrm{~b})$ ), we see that $a_{\epsilon}^{\prime \prime}(r)$ satisfies the second condition of the lemma.
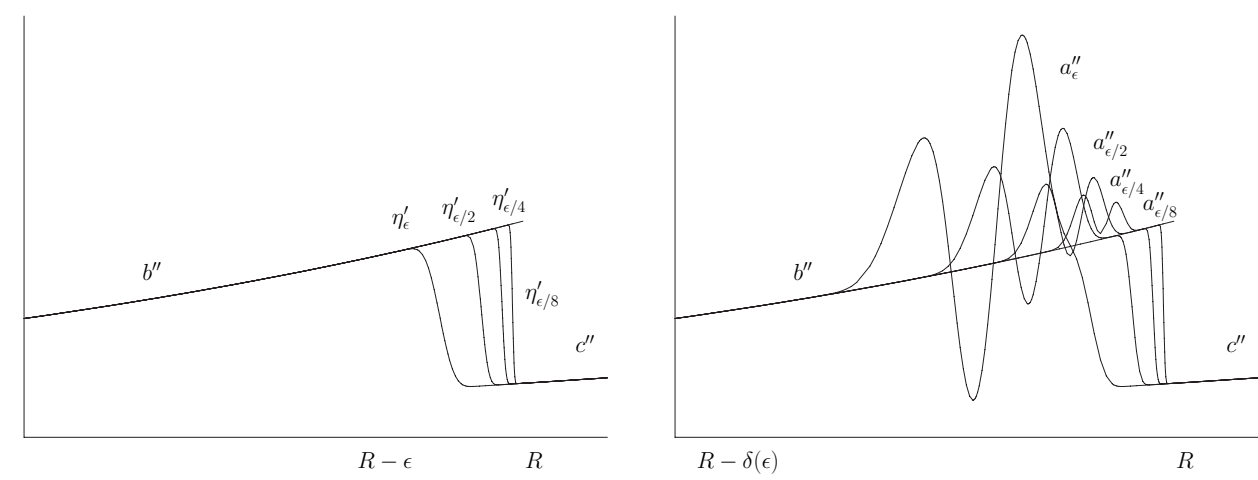

Figure 2: Interpolating between $b^{\prime \prime}$ and $c^{\prime \prime}$

Continuing with the proof of lemma 2.4, one may see by integrating that under the conditions of lemma 2.5, we have $a_{\epsilon}(r) \rightarrow a(r)$ and $a_{\epsilon}^{\prime}(r) \rightarrow a^{\prime}(r)$ uniformly as $\epsilon \rightarrow 0^{+}$(figure 3). For the functions $f(r)$ and $g(r)$ defining $\rho$, we can use lemma 2.5 to construct a sequence of functions $f_{\epsilon}(r)$ and $g_{\epsilon}(r)$ such that $f_{\epsilon}^{\prime}(r) \rightarrow f^{\prime}(r), f_{\epsilon}(r) \rightarrow f(r)$ as $\epsilon \rightarrow 0^{+}$, and $\lim _{\epsilon \rightarrow 0^{+}} \sup _{R-\delta(\epsilon)<r<R} f_{\epsilon}^{\prime \prime}(r)=f^{\prime \prime}(R)$. Similarly $g_{\epsilon}^{\prime}(r) \rightarrow g^{\prime}(r), g_{\epsilon}(r) \rightarrow g(r)$ and $\lim _{\epsilon \rightarrow 0^{+}} \sup _{R-\delta(\epsilon)<r<R} g_{\epsilon}^{\prime \prime}(r)=\cosh (R)$, where $\delta(\epsilon)$ comes from the construction in lemma 2.5. Moreover, for $\epsilon$ small enough, $g^{\prime \prime}(r)$ and $f^{\prime \prime}(r)>0$ for all $r$, so that $\rho_{\epsilon}$ is negatively curved for small $\epsilon$ (figure 2(b)). One may use these properties to show that $\sup f_{\epsilon}^{\prime \prime}(r) / f_{\epsilon}(r) \rightarrow$ $\operatorname{coth}^{2} R, \sup g_{\epsilon}^{\prime \prime}(r) / g_{\epsilon}(r) \rightarrow 1$, and $\sup f_{\epsilon}^{\prime}(r) g_{\epsilon}^{\prime}(r) /\left(f_{\epsilon}(r) g_{\epsilon}(r)\right) \rightarrow 1$ as $\epsilon \rightarrow 0^{+}$. From this, we deduce that $\lim _{\epsilon \rightarrow 0^{+}} k_{\epsilon}=\left(1+\operatorname{coth}^{2} R\right) / 2=\operatorname{coth} R \operatorname{coth} 2 R$. 


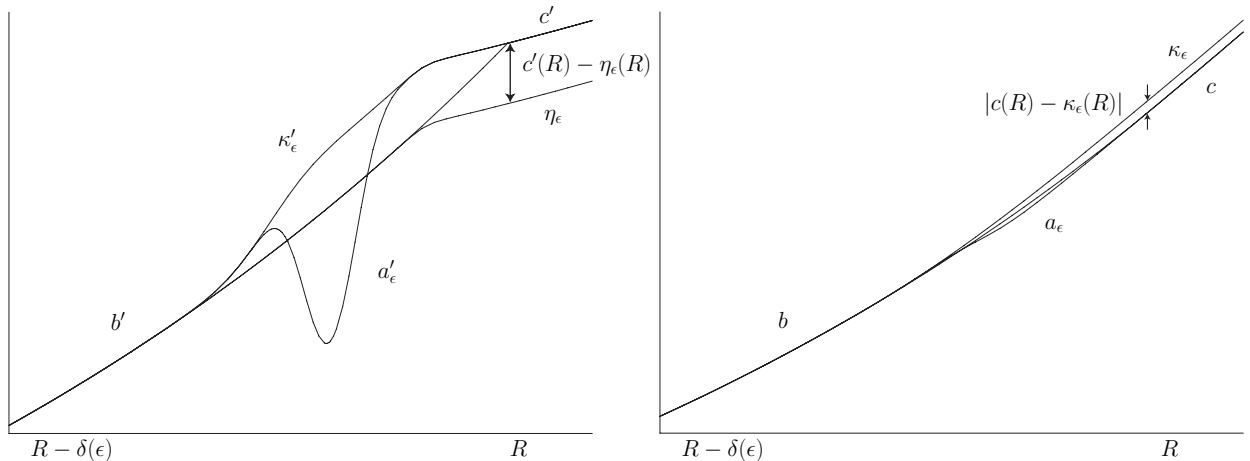

Figure 3: Smoothing $a^{\prime}$ and $a$

\section{Applications}

First, we state a theorem of Gabai, Meyerhoff, and Thurston.

Theorem 3.1 [8, 4.11] Let $\gamma$ be a minimal length geodesic in a closed orientable hyperbolic 3-manifold $M$, of length $l$ and tube radius $R$. Either

(1) $R>(\ln 3) / 2$, or

(2) $1.0953 / 2>R>1.0591 / 2$ and $l>1.059$, or

(3) $R=.8314 \ldots / 2$ and $M=V$ ol3, the closed manifold of third smallest volume in the Snappea census [17], which has volume $=1.0149 \ldots$.

The following corollary is of interest in controlling the geometry of a minimal volume hyperbolic 3-manifold. This improves on the estimate of theorem 1.1 of Gabai, Meyerhoff, and Milley in [7], and theorem 4.7 of Przeworski [14]. This estimate may be useful in a computational approach to find the minimal volume orientable hyperbolic 3-manifold proposed by Gabai, Meyerhoff, and Milley [7] by extending the computations and method of [8].

Corollary 3.2 The minimal volume orientable hyperbolic 3-manifold $M$ has $\operatorname{Vol}(M)>.32$, and the minimal length geodesic $\gamma$ in $M$ has tube radius $R<$ .956 .

Proof We may assume that $M$ is closed, since if $M$ had a cusp, then by Cao and Meyerhoff's result that a cusped oriented manifold has volume $>2.0298$ [5], which is greater than the volume of the Weeks manifold, $M$ would not have 
minimal volume. We may assume that $R>(\ln 3) / 2$, since in cases 2 and 3 of theorem 3.1, the volume is $>1.01$, which is greater than the volume of the Weeks manifold, as observed in [8].

By a result of Cao and Meyerhoff [5], $\operatorname{Vol}\left(M_{\gamma}\right)>2.0298$. Then by theorem 2.1, we have $\operatorname{Vol}(M)>\operatorname{Vol}\left(M_{\gamma}\right) /\left(\operatorname{coth}^{\frac{5}{2}}(\ln 3 / 2) \operatorname{coth}^{\frac{1}{2}}(\ln 3)\right)>.32$.

We also have

$2.0298<\operatorname{Vol}\left(M_{\gamma}\right) \leq(\operatorname{coth} R)^{\frac{5}{2}}(\operatorname{coth} 2 R)^{\frac{1}{2}} \operatorname{Vol}(M)<(\operatorname{coth} R)^{\frac{5}{2}}(\operatorname{coth} 2 R)^{\frac{1}{2}} .943$.

Thus, we get the upper bound $R<R_{0}<.956$, where

$$
2.0298=\left(\operatorname{coth} R_{0}\right)^{\frac{5}{2}}\left(\operatorname{coth} 2 R_{0}\right)^{\frac{1}{2}} .943 .
$$

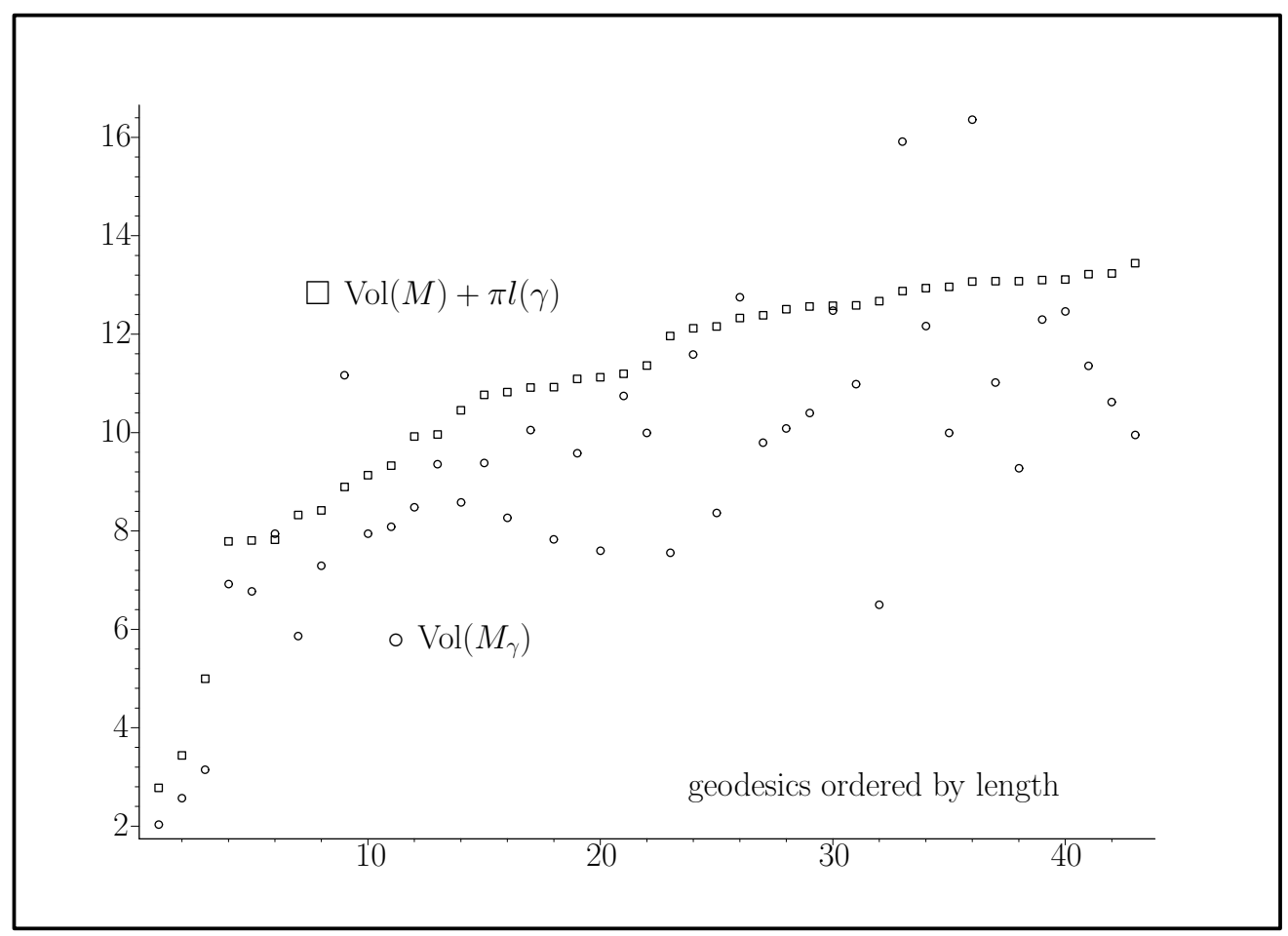

Figure 4: Bridgeman's conjecture for drilling geodesics in Weeks' manifold

Remark Przeworski has used theorem 2.1 and a result of Marshall and Martin [12] to show that the minimal length geodesic in a 3 -manifold of minimal 


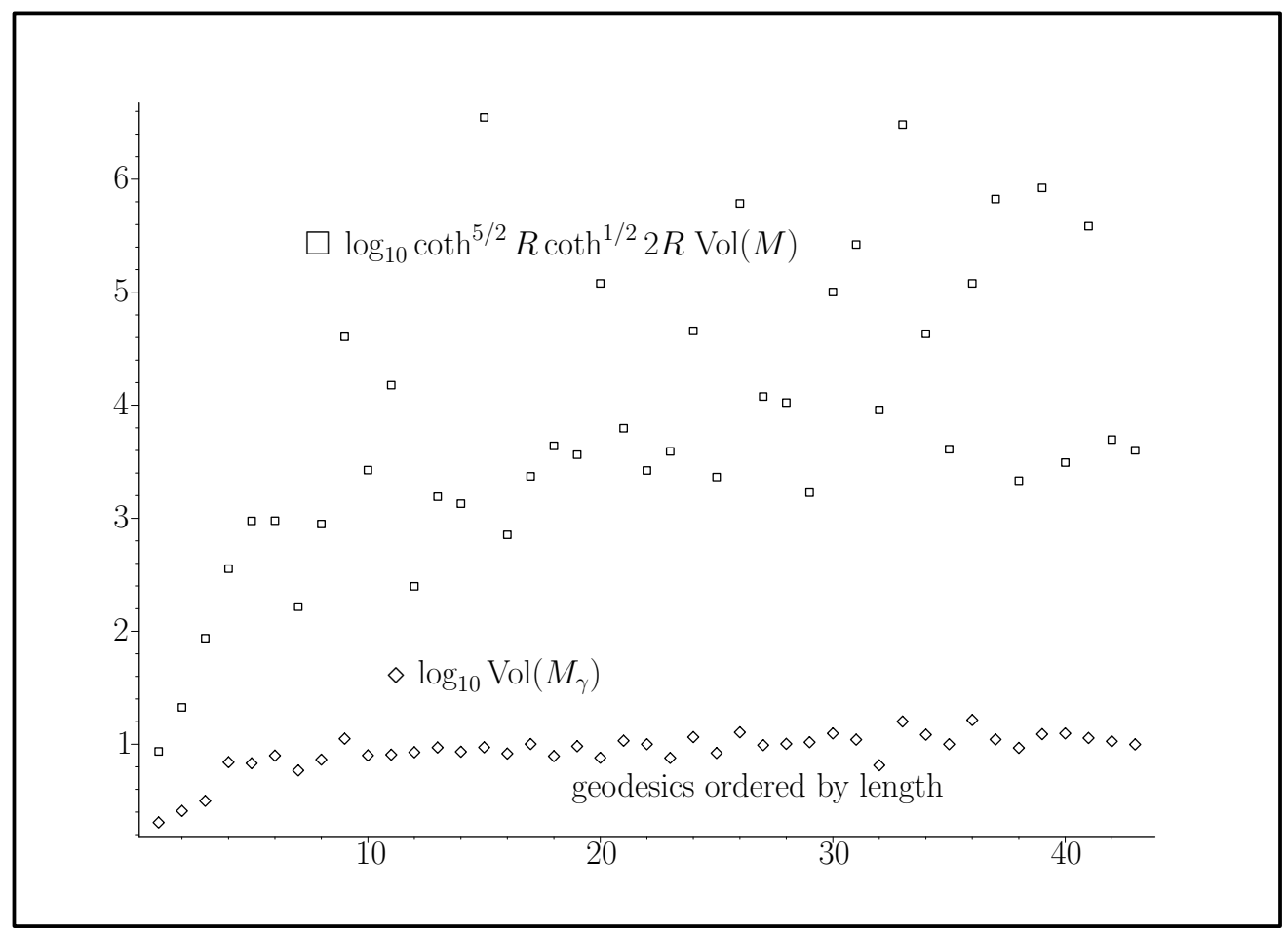

Figure 5: Log plot of the estimate for drilling geodesics in Weeks' manifold

volume is $\geq .184$ and has tube radius $R<.946$ (Proposition 7.4 [15]). Przeworski points out that this estimate could be improved slightly. He has also used theorem 2.1 and some packing arguments to improve the lower bound in theorem 3.2 to .3315 (Proposition 5.4, [16]).

It would be interesting to understand exactly how much the volume can increase when one drills out a geodesic. Bridgeman has conjectured [4] that if one drills an embedded geodesic $\gamma$ of length $l$ out of $M$, then $\operatorname{Vol}\left(M_{\gamma}\right) \leq \operatorname{Vol}(M)+\pi l$. Bridgeman proved this for many special examples. We have used Oliver Goodman's program tube [10] to give computational evidence that this conjecture is false in general. Figure 4 gives data obtained from tube, by drilling many of the shortest geodesics from the Weeks manifold. There appear to be five cases where Bridgeman's conjecture fails. The estimate given in theorem 2.1 is not at all close to being sharp, especially when $R$ is small, see figure 5 . 


\section{References}

[1] Gérard Besson, Gilles Courtois, Sylvestre Gallot, Lemme de Schwarz réel et applications géométriques, Acta Math. 183 (1999) 145-169

[2] Steven A Bleiler, Craig D Hodgson, Spherical Space Forms And Dehn Filling, Topology 35 (1996) 809-833

[3] Jeffrey Boland, Christopher Connell, Juan Souto, Volume rigidity for finite volume manifolds, preprint, http://www.math.uic.edu/ cconnell/papers.html

[4] Martin Bridgeman, Bounds on volume increase under Dehn drilling operations, Proc. London Math. Soc. (3) 77 (1998) 415-436

[5] Chun Cao, G Robert Meyerhoff, The orientable cusped hyperbolic 3manifolds of minimum volume, Invent. Math. 146 (2001) 451-478

[6] Ted Chinburg, Eduardo Friedman, Kerry N Jones, Alan W Reid, The arithmetic hyperbolic 3-manifold of smallest volume, Ann. Scuola Norm. Sup. Pisa Cl. Sci. (4) 30 (2001) 1-40

[7] David Gabai, G Robert Meyerhoff, Peter Milley, Volumes of tubes in hyperbolic 3-manifolds, J. Differential Geom. 57 (2001) 23-46

[8] David Gabai, Robert Meyerhoff, Nathaniel Thurston, Homotopy hyperbolic 3-manifolds are hyperbolic, to appear in Annals of Mathematics, http://www.math.caltech.edu/people/gabaipub.html

[9] Sylvestre Gallot, Dominique Hulin, Jacques Lafontaine, Riemannian geometry, second edition, Springer-Verlag, Berlin (1990)

[10] Oliver Goodman, tube, http://www.ms.unimelb.edu.au/〜snap/, tube comes with the program snap

[11] Sadayoshi Kojima, Deformations of hyperbolic 3-cone-manifolds, J. Differential Geom. 49 (1998) 469-516

[12] T Marshall, Gaven Martin, Volumes of hyperbolic 3-folds (2000), to appear in J. Conf. Geom. and Dynamics

[13] John W Morgan, On Thurston's uniformization theorem for three-dimensional manifolds, from: "The Smith conjecture (New York, 1979)", Academic Press, Orlando, FL (1984) 37-125

[14] Andrew Przeworski, Cones embedded in hyperbolic manifolds, J. Differential Geom. 58 (2001) 219-232

[15] Andrew Przeworski, Density of tube packings in hyperbolic space (2002), preprint, http://www.ma.utexas.edu/users/prez/

[16] Andrew Przeworski, A universal bound on density of tube packings in hyerbolic space (2002), preprint, http://www.ma.utexas.edu/users/prez/

[17] Jeff Weeks, SnapPea: A computer program for creating and studying hyperbolic 3-manifolds, available at http://www.northnet.org/weeks/ 